\title{
Semantic Cloud for Mobile Technology
}

\author{
Sonal Anand \\ B. Tech (VII Semester), BPIT \\ Rohini, Delhi, India
}

\author{
Sarvesh Gupta \\ B.Tech (VII Semester), BPIT \\ Rohini, Delhi, India
}

\author{
Shweta Fatnani \\ B.Tech (VII Semester), BPIT \\ Rohini, Delhi, India
}

\author{
Varsha Sharma \\ Lecturer, BPIT \\ Rohini, Delhi, India
}

\author{
Deepti Jain \\ Lecturer, BPIT \\ Rohini, Delhi, India
}

\begin{abstract}
The paper highlights and outlines the architecture of SCM (Semantic Cloud for Mobile), a blend of two emerging web technologies, semantic web and cloud computing, for revolutionizing data access and processing capabilities over mobile platform. The emphasis lies on decoupling of data processing and management from mobile hardware, alongwith the way SCM can be employed to resolve issues that have persisted over years, and exploring new opportunities that SCM may promise to offer.
\end{abstract}

Keywords: Semantic Web, Cloud Computing, Semantic Cloud for Mobile, SCM, Mobile Cloud, RDF, Semantic Cloud

\section{INTRODUCTION}

Semantic web is a mesh of information linked up in a way as to be easily processable by machines on a global scale. Semantic web is usually built on syntaxes using URIs, like http to represent data that can be held in databases or interchanged with www using RDF (Resource Description Framework) syntaxes. Semantic web also makes use of ontologies(by OWL) which enhance the web search, thus making the search program look for compact and more relevant content on web .

Cloud computing on the other hand refers to the computation done through Internet, i.e. allowing users to access database resources via Internet, without the need for maintenance of these resources, thus empowering the use of dynamic and scalable databases. Cloud computing provides the facility to access shared resources and common infrastructure, offering services on demand over the network to perform operations that meet changing business needs. The location of physical resources and devices being accessed are typically not known to the end user. It also provides facilities for users to develop, deploy and manage their applications 'on the cloud', which entails virtualization of resources that maintains and manages itself.

Mobile technology gives the power to access information anywhere and at any point of time. As the mobile phones guarantee portability, handleability, transportability, and better speed, its use as an information retrieval platform can promise the scope for a huge market.
Incorporating the former two technologies, i.e. the SEMANTIC CLOUD on mobile platform may cater the need for efficient, semantic access to cloud data on mobile.

\section{WHY THE TRIO?}

Statistics show that there were 415 million mobile phone users in India by March 2009 and nearly 117.82 million use mobile phone to access Internet. With the emergence of $3 \mathrm{G}$ and mobile Internet, there is an urgent requirement for IT support systems to provide mass storage space and fast computing capacity. The pace at which the mobile technology is spreading, the digital world is putting all its focus on development of and for mobile platform.

As the amount of data is expected to grow further and complexity is witnessing a consistent rise, it is better to reduce the dependency on mobile hardware.

Though the cost of hardware is decreasing, the decoupling of data storage, processing and management from mobile hardware will enhance performance, scalability and agility. The dependency on hardware can be reduced manifold by moving the data and processing on cloud, which offers high performance, low cost and high scalability features along with processing management and storage capability for mass data, without the hassle of worrying about mobile hardware configuration. Hosting on cloud also relieves user off the risk of losing important data due to system crash, hardware malfunctioning, virus attack, etc.

However, cloud computing faces a number of issues including privacy, data integrity, data security, authentication, identification of access rights, low information quality, ambiguity and design problems due to disparity in database development models, used by cloud providers. Here, semantic web comes to the rescue.

Semantic web provides a framework for control of cloud usage. The unified RDF can replace the disparate cloud database models. By using ontologies, semantic web aims at collecting structured information from web pages and redirect the information to client side to cater needs and preferences of the individual users. Semantic web also reduces cost and complexity of cloud computing by the use of rules laid down in 
RDF.

The issue of security, one of the major roadblocks in the success of cloud computing, is resolvable by a wide range of security mechanisms that the semantic web provides.

Figure 1 shows the suggested architecture of SCM.

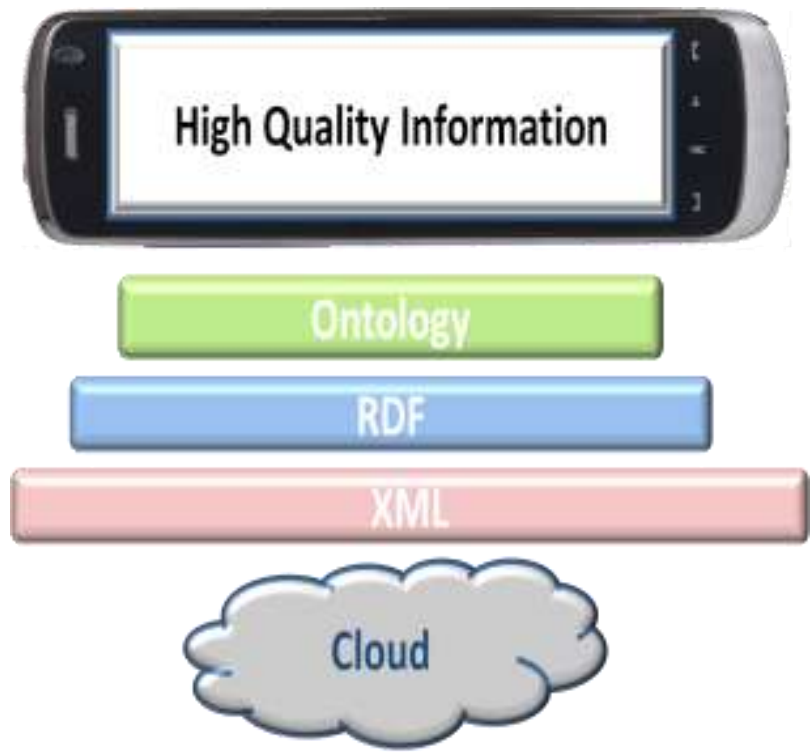

Figure 1) SCM Architecture

\section{Applications}

\section{M learning:}

Any sort of learning that happens when the learner is not at a fixed, predetermined location, or learning that happens when the learner takes advantage of the learning opportunities offered by mobile technologies. M-learning focuses on the mobility of the learner, interacting with portable technologies, and learning that reflects a focus on how society and its institutions can accommodate and support an increasing mobile population. Mobile learning can be used as a 'hook' to re-engage students
International Journal of Computer Applications (0975 - 8887)

Volume 8-No.12, October 2010

who seldom find time to engage in studying piles of books for the little knowledge they are concerned with. A major issue concerned with m-learning is content security or copyright issue from authoring group.

Hosting educational services on public cloud will guarantee equal access to all. For instance, use of SCM will provide the farmers with efficient and relevant access to farming updates and market scenario with content security being taken care of by the use of semantic web agents that deal with trust involving issues of semantics.

\section{E-Tourism:}

Mobile has lately been vital where the issue is to save time and effort of tourists worldwide, while searching for markets, restaurants, hotels and malls around any corner of the world. Putting e tourism data on mobile cloud may allow users to enjoy quick and efficient access to information on tourism products and services, at any point of time, regardless of where they are.The use of semantic web promises to provide relevant and compact information, by expanding choices offered to customers, by enabling drilling down to the level of desired detail, thus making the searching process a pleasure for them. Also important for authorities, is a simplified business process, with low operating cost and large market for tourism services, which Semantic Cloud for Mobile (SCM) promises to offer.

\section{M-Commerce:}

There have been a wide range of barriers regarding adoption of e-commerce in developing countries. These range from 'high cost of initial investment' to 'cost of system maintenance, to 'resistance to adoption of e-commerce'. While hosting on cloud will resolve cost and maintenance issues, m-commerce may take care of the small e-commerce market size, which is another concern for budding businessmen. Semantic web offers several security mechanisms ranging from XML Key Management System to validate certificates and signatures, to XML signature and XML encryption, which provide rules for encrypting or signing of business documents, thereby resolving the issue of 'resistance to adopt e commerce', which may be a matter of security and trust.

M-Health:

Following pie chart, figure 2, shows the statistics of awareness and adoption of $\mathrm{m}$-health according to a recent survey.

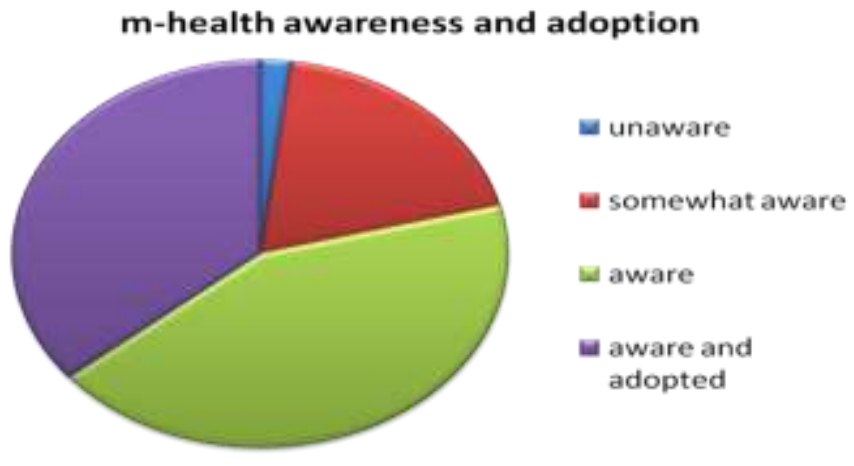

Figure 2) M-Health Adoption Statistics

The barriers in adoption of m-health may include the privacy issues, and the need for apt information about treatments and medicacies. Putting the data on mobile cloud, and offering a comfortable, semantic search experience to patients may relieve 
them off the time consuming, exhaustive search, while Xml encryption and Security Assertions Markup Language can take care of query privacy and data authentication respectively.

\section{NEW OPPORTUNITIES}

The goal of SCM is not confined to improvement in existing spheres but also to explore new possibilities. Two of these are listed below:

\subsection{University Search Engine}

While looking for a list of colleges on a famous search engine, we found innumerable results, with only a few of our concern. Out of these, most pointed to the respective campus websites, thus lacking the integration mechanism, which can collect and present the entire relevant information at one place.
International Journal of Computer Applications (0975 - 8887)

Volume 8-No.12, October 2010

A search engine, based on semantic cloud, if accessible to students and faculty, through their mobile phones, can provide them, with the ability to experience relevant, focussed and fast access to an integrated information of campuses across the world, including locations, faculty, management, canteen, library, hostels and the like, with drilled down details as per their choice of search.

For instance, a candidate wants to search for placement details of his favorite college prior to admission process. The search engine requires offering drill down to the deepest level of search, from locations to college names to details of each college. Figure 3) shows the possible browsing experience. As the candidate searches for the list of colleges, he gets to choose between different locations. After selecting a campus location, the entire list of colleges in the area is displayed. As the candidate selects a college, he can select and view the information related to his interest.

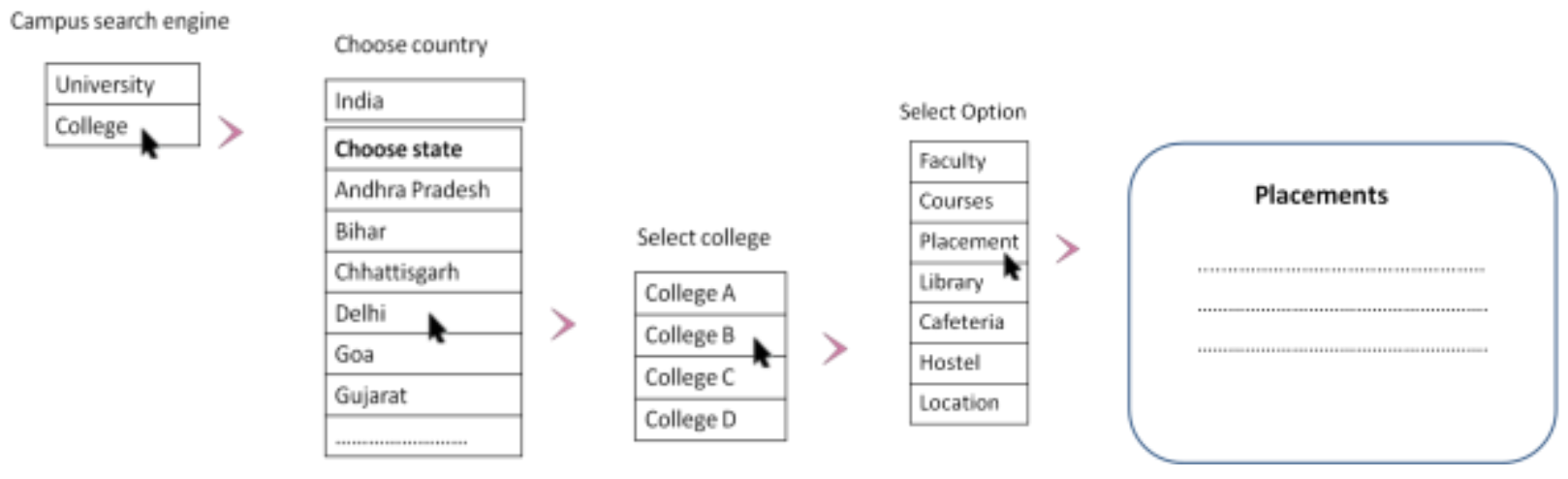

Figure 3) University Search Engine

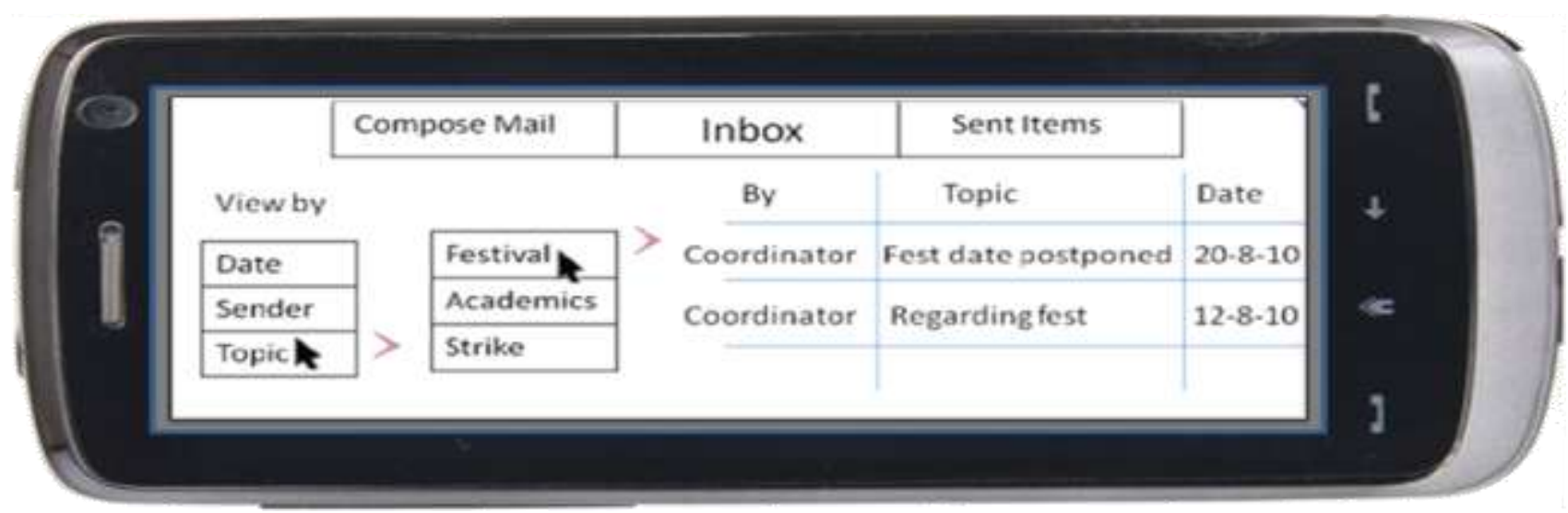

Figure 4) Campus Mailing Server 
4.2 Campus Mailing Server

A mailing server, when implemented on semantic cloud, and offered to students and faculty on their mobile phones, inside a campus, may provide a better medium of communication regarding admissions, examinations, infrastructure, faculty, fests etc. The server may provide a centralised platform, from where all registered members can send and receive messages. Whenever the need for communication arises, for instance when faculty wants a message to be conveyed to all, an instant messaging system is required, that can broadcast, multicast or unicast information to intended recipients. A possible interface is depicted in figure 4 . Such a mailing server can reduce time and effort of the recipient as he looks for a mail inside the inbox, by extending a wide range of search choices to him regarding subjects, sender, date and time of delivery.

\section{Conclusion}

SCM application is usable on any mobile device, which supports a suitable browser, independent of its operating system and hardware configurations. Besides this, SCM also guarantees low cost of deployment and development. Features like Html5 allow application to continue even if internet connection fails. SCM promises to offer a huge market with large revenue for developers, as the percentage of mobile Internet users seems to escalate exponentially with time.

\section{REFERENCES}

[1] Berners-Lee, Tim; James Hendler and Ora Lassila (May 17, 2001). "The Semantic Web". Scientific American Magazine. http://www.sciam.com/article.cfm?id=the-semantic web\&print=true. Retrieved March 26, 2008
International Journal of Computer Applications (0975 - 8887)

Volume 8-No.12, October 2010

[2] Srinivasa Rao V, Nageswara Rao N K, E Kusuma Kumari."Cloud Computing: An Overview", Journal of Theoretical and Applied Information Technology (Vol 9. No. 1 - 2009) http://www.jatit.org/volumes/research papers/Vol9No1/10Vol9No1.pdf

[3] "Mobile Internet has high potential in India", http://www.ciol.com/News/News-Reports/Mobile-Internet-hashigh-potential-in-India/21709122569/0/

[4] Wikipedia, http://en.wikipedia.org/wiki/MLearning

[5] Abbie Barbir,"Web Services Security: An Enabler of Semantic Web Services" http://www.cs.unb.ca/baseweb/baseweb03/papers/abbie-barbirBaseWeb2003-paper1.pdf

[6] Kevin Green,'Tripple Tree MHealth Research and Survey Report" tree.com/ResearchRequest. aspx ?researchId $=46$ http://www.triple-

[7] IBM Point of View: Security And Cloud Computing Cloud Computing, White Paper November 2009

[8] Graham Klyne, Semantic Web Applications http://www.ninebynine.net/Papers/SemanticWebApplications.df

[9] Why Cloud Computing is the Future of Mobile, http://www.readwriteweb.com/archives/why_cloud_computin_i s_the_future_of_mobile.php 